\title{
Appropriation d'une stratégie opératoire : un enjeu du collectif de travail
}

Developing and adapting operative strategies: a key issue for work teams

\section{Lucie Cuvelier et Sandrine Caroly}

\section{(2) OpenEdition}

\section{Journals}

Édition électronique

URL : http://journals.openedition.org/activites/2249

DOI : $10.4000 /$ activites.2249

ISSN : 1765-2723

\section{Éditeur}

ARPACT - Association Recherches et Pratiques sur les ACTivités

\section{Référence électronique}

Lucie Cuvelier et Sandrine Caroly, «Appropriation d'une stratégie opératoire : un enjeu du collectif de travail », Activités [En ligne], 6-2 I octobre 2009, mis en ligne le 15 octobre 2009, consulté le 19 avril 2019. URL : http://journals.openedition.org/activites/2249; DOI : 10.4000/activites.2249

\section{(ब) $(1 \Theta \Theta$}

Activités est mis à disposition selon les termes de la licence Creative Commons Attribution - Pas d'Utilisation Commerciale - Pas de Modification 4.0 International. 


\title{
Appropriation d'une stratégie opératoire : un enjeu du collectif de travail
}

\author{
Lucie Cuvelier \\ Cnam, Centre de Recherche «Travail \& Développement », Equipe Ergonomie, \\ 41 rue Gay Lussac, 75005 Paris, France. \\ Lucie.cuvelier@cnam.fr
}

Sandrine Caroly

Université Pierre Mendès France, PACTE, Le patio, BP 47, 38040 Grenoble cedex 09 Sandrine.caroly@upmf-grenoble.fr

\begin{abstract}
Developing and adapting operative strategies: a key issue for work teams. This research is based on the monitoring of an important organizational and technical change conducted in a sorting office. The aim is to understand how postal workers are developing their activities following both the organizational changes and the introduction of new technical systems. Research from various fields of sociology and educational science led us to approach the ergonomic analysis of this change through the concept of "appropriation", which we decline on three levels: the appropriation of tools, the appropriation of procedures and the appropriation of "professional actions". The development of an instrument (from the "sorting tool" artefact) and the appropriation of a new strategy used for sorting post are analysed more specifically. The results show that this appropriation is in several ways dependent on the requirements of each work situation and on performance objectives pursued by operators, but that it is also dependent on collective work.
\end{abstract}

KEYWORDS

Appropriation, collective work, change, design, development.

\section{1.- Introduction}

«Nous vivons une époque où les entreprises publiques et privées se transforment en profondeur et à un rythme accéléré » (Sardas, 1994). Dans certains cas, les changements menés touchent tant d'aspects à la fois - espaces, dispositifs techniques, outils, organisations, valeurs et sens du métier qu'ils instaurent une véritable « coupure », une bascule vers un nouveau système. Il en résulte pour les opérateurs des transformations dans la réalisation de leur travail, nécessitant le développement de nouvelles compétences et la reconstruction de leur activité (Lacoste, 1992). L'originalité de notre recherche, menée dans un centre de tri et de distribution du courrier, est d'être focalisée sur un changement organisationnel et technique majeur en cours de réalisation, de le suivre dans sa mise en œuvre. Son objectif est de comprendre comment, durant les premiers mois suivant un tel changement, les opérateurs reconstruisent leur activité dans le double contexte d'une organisation du travail modifiée et de l'implantation de nouveaux dispositifs techniques : comment s'approprient-ils les nouveaux outils et les nouvelles procédures de travail ? Quels sont les éléments à même de faciliter ou au contraire d'entraver ces processus d'appropriation? Quelles sont les modalités qui sous tendent le développement de nouveaux gestes professionnels?

Pour appréhender la problématique du changement dans les entreprises, Bernoux (2002) propose de s'appuyer sur le concept d' « appropriation » qu'il développe en sociologie. En effet, pour cet auteur, «les acteurs [...] ne sont jamais passifs, [...] sans leur implication et sans l'appropriation des outils, 
les changements ne peuvent tout simplement pas avoir lieu» (Bernoux, 2004, p.9). Il propose alors une définition du concept d'appropriation centrée sur « la maitrise de l'action de travail par celui qui l'exécute, » « le sens donné au travail » par l'opérateur et « les modifications induites [par les acteurs] dans ce qui avait été prévu par les concepteurs » (Bernoux, 2004, pp.56-57). Cette définition nous apparaît cohérente avec les bases constituées de l'approche ergonomique centrée sur l'activité. Mais elle ne rend pas compte des dimensions développementales de l'activité.

Afin d'appréhender ces processus d'appropriation dans le cadre de notre recherche en ergonomie, nous déclinons cette notion sous trois angles : l'appropriation des outils, l'appropriation des procédures et l'appropriation des gestes de travail. Puis, sur la base des méthodes présentées, nous mettons en évidence les modalités d'appropriation d'une stratégie opératoire développée lors d'une nouvelle phase de tri du courrier. Parmi ces modalités, nous accordons une place particulière aux dimensions collectives du travail. En effet, ce contexte de changement global, au cours duquel toute l'équipe doit faire face en même temps à une nouvelle situation de travail, est un cadre méthodologique opportun pour préciser le rôle que peut jouer le collectif dans l'appropriation de stratégies opératoires.

\section{2.- La notion d'appropriation}

\section{1.- Appropriation des outils et genèse instrumentale}

Afin de rendre compte du fait que toute nouveauté technique s'inscrit dans un milieu social, cognitif ou culturel qui la précède, dans des «manières de faire » déjà établies, Béguin (2007) propose un modèle de l'appropriation basé sur la théorie instrumentale développée par Rabardel (1995). Selon cette théorie (Rabardel, 1995), l'instrument est une unité mixte constituée d'un artefact (objet fabriqué de nature matériel ou symbolique) et d'une composante liée à l'action, nommée « schèmes d'utilisation », «formée d'invariants organisateurs de l'activité du sujet ». (Rabardel, 2005, p.14). L'appropriation des outils est alors abordée sous l'approche développementale de la construction des instruments. Ces « genèses instrumentales », qui « constituent une conceptualisation, au plan des instruments, des processus d'appropriation» (Béguin, 2005, p.39) peuvent être orientées vers le sujet - instrumentation - ou vers l'artefact - instrumentalisation. Dans la perspective développementale de l'activité médiatisée proposée par Rabardel (1995), «l'appropriation des outils culturels, des usages et développement des instruments et des individus » est l'unité d'analyse des activités constructives (Folcher \& Rabardel, 2004, p.254) c'est-à-dire des activités orientées vers la constitution, par le sujet, des ressources de son action et de son développement.

Ainsi les genèses instrumentales témoignent de l'appropriation. "Elles supposent également une dimension plus large de l'activité [...] : les genèses professionnelles » (Béguin, 2007, p.372). Ces dernières « concernent le développement par le sujet des ressources de son action: la constitution des instruments [...] mais aussi de beaucoup d'autres aspects, tels que les développements des conceptualisations et compétences, ainsi que des formes organisées de l'action au sein des collectifs » (Op. Cit.). Dans la situation de transformation brutale du système de travail qui nous intéresse, d'autres dimensions de l'activité sont effectivement convoquées: en développant des instruments, les opérateurs s'approprient aussi de nouvelles procédures, et à l'interface des prescriptions et des nouveaux dispositifs techniques de travail, ils développent de nouvelles compétences et construisent de nouveaux gestes professionnels.

\section{2.- Appropriation des procédures et application de la prescription}

L'approche instrumentale décrite au paragraphe précédent concerne aussi bien des artefacts matériels que symboliques, tels les règles, consignes ou procédures. Le modèle de l'appropriation précédemment exposé peut donc inclure les prescriptions «qui représentent en effet une forme particulière d'instrument » (Mayen \& Vidal-Gomel, 2005). C'est ce que propose Vidal-Gomel (2002) dans une 
étude sur le rapport aux règles de sécurité dans le domaine de la prévention des risques électriques. "En considérant que les règlements ou les règles de sécurité sont des artefacts prescriptifs, [...] nous soulignons d'une part que les instruments qu'ils permettent de constituer ne sont pas donnés d'emblée et qu'il ne s'agit pas uniquement d' "appliquer », [...] [et] d'autre part, [qu'] un artefact prescriptif peut donner lieu à plusieurs instruments » (Mayen \& Vidal-Gomel, 2005, p.115). De façon plus classique en ergonomie, le rapport à la prescription relève de l'écart entre la « tâche prescrite » (tâche conçue par celui qui en commande l'exécution) et la «tâche effective » (résultat d'un apprentissage de l'opérateur) (Leplat \& Hoc, 1983). Cette dernière recouvre la « tâche comprise », c'est-à-dire ce que l'opérateur pense qu'on lui demande de faire (en fonction de l'intelligibilité des consignes et de ses propres modèles) et la «tâche appropriée », c'est-à-dire spécifiée par l'opérateur à partir de la tâche comprise et estimée par lui plus adéquate en fonction de ses propres valeurs, de ses priorités, etc. (Veyrac Merad-Boudia, 1998).

Les approches de la didactique professionnelle questionnent le rapport aux règles ${ }^{1}$, sous l'angle des compétences et de leur développement. Ainsi, Mayen et Savoyant (1999) proposent quatre niveaux de rapport aux règles qui « pourraient constituer quatre étapes de tous processus d'appropriation des règles »:

— « Respect de la règle par absence de doute;

- Remise en cause de la règle au profit de la référence à ses propres perceptions, à son propre raisonnement; à sa propre capacité d'initiative (et revendiquant une part de liberté individuelle) ;

- Respect de la règle reconnue et réinventée dans sa nécessité logique et certitude du bien fondé de son action ;

- Discussion de la règle après l'action, éventuellement pour la remettre en cause et participer à son évolution » (Mayen \& Savoyant, 1999, p.90).

Pour ces auteurs, l'application des procédures « comporte toujours un choix » et constitue une activité à part entière. Ce choix de respecter des procédures suppose une connaissance des causes et des principes qui fondent les règles ainsi que de tous les éléments qui permettent de comprendre les liens entre les différentes procédures. Pourtant, bien souvent, cette « théorie » fait défaut dans les prescriptions et les formations. Et cette absence de justification explicite des procédures ne facilite pas leur «appropriation [qui] est indissociable de la conceptualisation » (Op. cit.). En ergonomie, une attention particulière est portée sur les processus de réélaboration des règles (Caroly, 2002 ; Gonzalès, Teiger, \& Weill-Fassina, 2002). Ils traduisent des phénomènes d'apprentissage, d'appropriation mais aussi de transmission et de développement de l'activité.

\section{3.- Appropriation des gestes et développement des compétences}

Dépendante de l'usage des outils et de la prescription, l'appropriation d'une nouvelle gestuelle par les opérateurs est le troisième angle sous lequel nous pouvons aborder l'analyse du changement en cours. Le geste, « système complet » intégrant des composantes biomécaniques, cognitives et psychiques, est un « compromis entre les objectifs, la tâche, les moyens de travail et les caractéristiques individuelles de celui qui les réalise » (Bourgeois, Lemarchand, Hubault, Brun, Polin, Faucheux, et al., 2006, p.163). En psychologie, « on a coutume en général de référer la formation du geste à la manière dont M. Mauss l'inscrivait dans une tradition » (Clot, 1999). Pour ce sociologue, la technique, au sens précis d' « acte traditionnel efficace » qu'il donne à cette notion (Mauss, 1934; cité par Clot, 1999), ne concerne pas que l'usage d'instruments, d'outils ou de machines mais aussi l'usage du corps. Ces « techniques du corps » sont transmises, apprises, en lien étroit avec la culture (Dejours, Dessors \& Molinier, 1994). Et cet apprentissage demande du temps car l'appropriation d'une

1. Il s'agit dans ces études plus particulièrement de « règles de sécurité » mais les auteurs notent que d'autres cas amènent à penser que les conclusions faites dans le domaine de la gestion des risques peuvent concerner un plus grand nombre de situations. 
gestuelle relève aussi de l'expérience. Y. Clot souligne que pour qu'un geste " soit effectivement approprié par moi - et il faut du temps et des échecs - il faut qu'il devienne approprié pour moi. [...] Un geste vraiment transmis, c'est-à-dire approprié, n'est plus tout à fait le même geste » (Clot, 1999, p.2-3). Il est reconstruit, transformé, convertis en « ressource interne de son propre développement ». Les psychologues ont alors étendu le concept d'instrument développé par Rabardel (1995), au corps considéré comme un «hybride », «exactement comme l'est l'artefact [auquel] le sujet doit attribuer la fonction de moyen adapté en vue des fins qu'il poursuit » (Fernandez, 2001, p.31).

En ergonomie, l'appropriation d'un geste est généralement abordée sous l'angle de la « construction des gestuelles » (Chassaing, 2004) ou du « développement des compétences » (Weill-Fassina \& Pastré, 2004). Différents auteurs ont ainsi étudié la construction des compétences à travers l'analyse des modalités de régulations individuelles et collectives mises en œuvre par les opérateurs (Gaudart \& Pueyo, 2000 ; Flageul-Caroly, 2001). D’une façon générale, les résultats de ces recherches mettent en évidence les objectifs des stratégies gestuelles des opérateurs et font référence à l'expérience pour expliciter leur développement. K. Chassaing (2005), par exemple, analyse les dynamiques de construction des gestuelles en retraçant l'histoire des gestes. Elle met ainsi en lumière la signification attribuée à ces gestuelles par les opérateurs et identifie des " déterminants des stratégies de travail selon les parcours antérieurs des uns et des autres» (Chassaing, 2004). Ces différents travaux nous révèlent donc que l'opérateur a un rôle actif dans la construction de ses compétences et dans l'appropriation de stratégies gestuelles. Ces compétences variées se développent au fil du temps et relèvent de divers registres d'expérience.

\section{4.- Appropriation et dimensions collectives du travail}

Les concepts exposés précédemment font tous référence, de façon plus ou moins explicite, à des aspects collectifs, partagés du travail. Dans l'approche instrumentale la notion de « genèse professionnelle » proposée par Béguin intègre une dimension collective (Béguin, 2007) et le développement du sujet tient compte de l'histoire « des communautés, collectivités et groupes sociaux d'appartenance et de vie » (Rabardel, 2005, p.13). Du point de vue de l'appropriation des prescriptions, les auteurs cités évoquent aussi l'importance des phénomènes collectifs dans le rapport de l'individu à la règle. Mayen et Vidal-Gomel (2005) ont pu observer en formation comme en situation de travail que «la remise en cause de la règle, sa mise en délibération, les différentes utilisations d'une règle et l'existence de moyens complémentaires revêtent un caractère collectif, partagé » (Mayen \& Vidal-Gomel, 2005, p.125). De même, à l'issue d'une analyse comparative entre deux bureaux de poste, Caroly montre que la réélaboration des règles n'est pas le seul fait d'une redéfinition individuelle. «Dans le bureau de province, l'activité collective, associée avec un collectif de travail, a abouti à la réélaboration des règles. Ce n'est pas le cas dans le bureau de banlieue où le collectif de travail n'est pas constitué » (Flageul-Caroly, 2001, p.213). Enfin, les gestes constituent « une arène sociale où se mesurent les manières de voir, de sentir et de faire » (Clot, 1999, p.3) et le regard porté par les «Autres » sur les gestes mis en œuvre par chacun est « constitutif » du système de reconnaissance dans lequel agissent les opérateurs (Dejours, Dessors, \& Molinier 1994 ; Bourgeois et al, 2006). Le développement d'un geste se fait «toujours dans un mouvement partagé avec autrui, échangé, adressé » (Clot \& Fernandez, 2005, p.73). Sa construction relève notamment de l'expérience du travail des autres: par exemple, «les opérateurs s'inspirent des façons de faire des opérateurs qu'ils jugent compétents $\gg$ (Chassaing, 2004). Elle tient aussi compte des ressources et contraintes offertes par les autres opérateurs dans le cadre du travail collectif: "la construction de compétences multifonctionnelles [...] peut découler d'une régulation collective » telle une répartition particulière des tâches au sein de l'équipe (Gaudart \& Weill-Fassina, 1999, p. 56).

Ainsi, le pôle «Autres » est bien présent sous des formes diverses, dans les trois approches de l'appropriation proposées, ce qui nécessite de préciser le cadre de référence dans lequel nous positionnons notre recherche (Benchekroun \& Weill-Fassina, 2000). Même si, les concepts sont encore loin d'être stabilisée en ergonomie (Flageul-Caroly, 2001), nous appuyons nos analyses sur la distinction 
entre le «travail collectif » et le « collectif de travail » (Benchekroun \& Weill-Fassina, 2000; Flageul-Caroly, 2001; Caroly \& Clot, 2004). Le travail collectif correspond à la manière de travailler ensemble, de coopérer, de collaborer, de s'entraider (Schmidt, 1994; De la Garza \& Weill-Fassina, 2000). Le collectif de travail désigne le « genre professionnel », la manière dont les professionnels définissent leur rapport aux objets et aux autres, ce qu'ils s'autorisent de faire dans les relations sociales et ce qu'ils s'empêchent de faire (Clot, 2004). C'est aussi un système de valeurs de référence au métier exercé, différent du référentiel opératif commun qui est indispensable pour le travail collectif. Pour qu'il y ait collectif de travail, «il faut simultanément plusieurs travailleurs, une œuvre commune, un langage commun, des règles de métier, un respect durable de la règle par chacun, ce qui suppose un cheminement individuel qui va de la connaissance des règles à leur intériorisation » (Cru, 1988). Ces règles dites « de métier » sont à différencier des règlements, procédures, normes ou consignes applicables sur les lieux de travail.

\section{3.- Méthodes}

\section{1.- Description du terrain de recherche}

Afin d'améliorer le service rendu aux clients et d'accroître la productivité, une succession de réformes d'une ampleur inédite est menée au sein l'entreprise dans laquelle nous sommes intervenus. À l'origine de cette modernisation se trouve un processus d'ouverture à la concurrence engagé depuis le début des années 1990. Dans ce contexte, la demande à laquelle nous avons répondu était portée par la Médecine de Prévention Professionnelle qui s'interrogeait sur les liens potentiels entre les changements menés et la santé des travailleurs. Au-delà de l'évaluation de l'impact des changements sur la santé des opérateurs, il s'agissait de comprendre comment ces derniers reconstruisent leur activité face aux évolutions organisationnelles et techniques.

Le changement que nous avons suivi lors cette recherche concerne un centre de tri et de distribution du courrier sur lequel travaillent 37 facteurs. Il présente la particularité d'avoir été mené en une seule étape. Du jour au lendemain, l'équipe et son encadrement ont déménagé dans de nouveaux locaux et une nouvelle organisation du travail a été mise en place. À l'origine de ces changements, se trouve la mécanisation du courrier avec, notamment, l'implantation d'une nouvelle machine capable de trier les plis directement dans l'ordre de « la tournée du facteur » c'est-à-dire dans l'ordre de la distribution. Le « temps de tri » est donc diminué au profit du « temps de distribution », puisque désormais, seules les lettres de format «non standard» (lettres trop petites ou trop grandes, presse, lettres non lues par les machines...) continuent d'être triées à la main. Quelques tournées ont été supprimées tandis que la durée de chaque tournée a été augmentée (ceci engendrant d'autres répercussions sur l'activité de travail telle l'évolution des moyens de locomotion: passage du vélo au scooter par exemple). En parallèle, pour le tri de ces lettres «non standard » un nouveau casier de tri manuel a été introduit. Son principal aspect novateur par rapport aux anciens casiers est l'intégration de cases verticales permettant d'accroître le nombre de séparations sur un espace de tri restreint. Sur ce nouveau casier, chaque case correspond à un point de distribution (et non plus à une rue ou à un pâté de maison). Le tri manuel est donc réalisé en une seule étape, « boite aux lettres par boîte aux lettres », directement dans l'ordre de la tournée. Autrefois le tri de la tournée était composé de deux étapes: le piquage et le coupage, qui sont en réalité intiment liés pour anticiper la tournée (Clot, Scheller, Caroly, Millanvoye, \& Volkoff, 2000). La suppression de ces deux étapes par les organisateurs a permis d'augmenter la productivité en diminuant le nombre de manipulations des plis.

\section{2.- Choix de la situation de travail : «la fusion »}

À l'interface de l'introduction de la machine et du casier de tri apparaît une nouvelle étape de travail, nommée « la fusion ». En effet, à l'issue du tri, le facteur se retrouve désormais avec deux lots de courrier triés dans l'ordre de sa tournée: 
— Les lettres «standard », qui ont été triées mécaniquement par la machine et disposées dans des caissettes ;

— Les lettres « non standard », triées manuellement dans le casier.

Il doit alors « fusionner » ces deux lots en un seul avant de partir en distribution. Étape peu visible avant le changement sur le site observé comme au niveau national (absente dans les tests, peu présente dans les communications observées et dans les entretiens réalisés....), « la fusion » se retrouve dès le premier jour du changement au centre de débats à tous les niveaux hiérarchiques de l'établissement et interroge l'ergonome du fait de la variabilité des stratégies opératoires développées par les opérateurs. C'est donc sur cette étape que nous nous sommes focalisés pour étudier l'appropriation. Par la suite, cette focale a été validée par les différents acteurs et s'est révélée féconde dans le sens où elle suscitait des « débats de métier » et des échanges sur le travail permettant de dépasser le climat revendicatif.

\section{3.- Techniques d'analyse}

Des observations ouvertes et systématiques ont été menées avant et après le changement, sur les phases de tri et de distribution. Le choix de l'échantillon de facteurs observés (15 opérateurs) s'est fait sur la base du volontariat et selon des critères d'âge, de sexe ainsi qu'en fonction du type de tournée réalisée. La prise en compte de la dimension collective s'est faite à la fois lors des observations systématiques et lors d'observations plus libres sur la structuration du groupe. Nous avons notamment réalisé régulièrement (environ une fois par mois) des « cartographies » des stratégies développées lors de la « fusion » par l'ensemble de l'équipe afin de pouvoir croiser les observations avec les caractéristiques des situations de travail, celles des individus, et celles du collectif.

En plus d'apporter des connaissances sur l'activité réelle, ces observations ont servi de supports pour produire des explicitations de la part des opérateurs. Des entretiens individuels ont été conduits de façon plutôt informelle, généralement durant la phase de distribution (pauses au café par exemple). Les données ont été recueillies par une prise de note manuelle. Dans un deuxième temps, deux entretiens collectifs, ont été menés. Les participants ( 8 facteurs « invités ») ont été choisis en fonction des résultats des observations réalisées précédemment (diversité des techniques de fusion développées). La première réunion était un entretien collectif de co-analyse des premiers résultats. Son objectif était d'identifier comment, à travers les échanges dans l'équipe, les contraintes liées au changement avaient été intégrées et s'inséraient dans une redéfinition collective du travail. Le second entretien a été réalisé sur la base des techniques d'auto-confrontation et d'allo-confrontation collective à partir de films de l'activité de trois opérateurs volontaires (Mollo \& Falzon, 2004). Ces entretiens collectifs ont duré $1 \mathrm{~h} 30$ et 2 heures chacun. Ils ont été enregistrés puis retranscrits intégralement. Les verbalisations ont ensuite fait l'objet d'une analyse de contenu fondée sur un traitement thématique.

L'analyse de la conduite du changement et de la conception des dispositifs de la nouvelle situation de travail est principalement fondée sur le recueil des documents et des traces: documents diffusés sur le site, études menées sur les « innovations technologiques », plannings diffusés au niveau national... Ce recueil a été complété par plusieurs entretiens avec des « acteurs clefs » de l'entreprise ayant participé à ce changement (Directrice des Ressources Humaines, Directeur Organisationnel et Technique du Courrier, Directeur de Groupement, chargé de mission Prévention des Risques...). 


\section{4.- Résultats}

\section{1.- Usage du casier lors de la fusion}

\subsection{1.- Appropriation de l'artefact}

Dès le premier jour du changement, deux principales stratégies ${ }^{2}$ de fusion semblent s'imposer sur le site:

- Soit les facteurs utilisent un «sabot », outil métallique constitué de deux pièces verticales servant à maintenir les plis. Le lot trié par la machine y est positionné « sur la tranche » afin de faire défiler les lettres, d'en lire l'adresse et d'y intercaler les plis triés manuellement, préalablement « décasés ». Les facteurs effectuent cette opération debout ou assis. Nous appellerons cette stratégie la «stratégie du sabot».

- Soit les facteurs insèrent les plis triés par la machine dans le casier. Il s'agit ici d'une genèse instrumentale, et plus précisément d'une instrumentalisation puisque les opérateurs attribuent une nouvelle fonction (fonction constituée: la fusion) à l'artefact « casier » dont la fonction constituante était uniquement de classer les lettres non mécanisables. Nous appellerons cette stratégie « la stratégie du casier».

Ces « différentes manières de faire la fusion » correspondent à la mobilisation de différents schèmes orientés vers l'objet de l'activité (les lettres à trier) et dont la signification est donnée par l'acte global de la fusion du courrier. Ce sont donc des schèmes d'action instrumentée (Rabardel, 1995). Ils sont mis en œuvre par les opérateurs dans « une situation vraiment nouvelle » puisqu'elle implique l'utilisation de nouveaux artefacts (le casier et le sabot) et vise des objets nouveaux (les lettres mécanisées) auxquelles de nouvelles transformations doivent être appliquées (la fusion). Ces nouvelles compositions de schèmes, qui permettent « la maitrise de la nouvelle situation », sont produites progressivement par transformation, réorganisation, fragmention et recomposition de schèmes disponibles (Op. Cit.). L'utilisation fréquente par certains facteurs du terme « billot » à la place du mot « sabot » peut illustrer cette constitution d'une nouvelle composition de schèmes à partir de schèmes disponibles. Les « billots » étaient des cales de bois utilisées autrefois par quelques facteurs pour maintenir le courrier verticalement. L'emploi de ce synonyme suggère que le schème d'action instrumenté mis en œuvre lors de l'utilisation du sabot soit lié au schème développé sur cet ancien outil (maintenir des lettres verticales) par extension des classes de situation (fusionner), des objets traiter (lettres mécanisées) et des artefacts (le sabot).

\subsection{2.- Appropriation de la procédure de fusion prescrite}

La mobilisation de ces différents schèmes d'utilisation et les controverses que cela suscite au sein de l'équipe interrogent sur la procédure prescrite. Sur le terrain, à tous les niveaux hiérarchiques (facteurs, facteurs de secteur ${ }^{3}$, encadrement de proximité), la «tâche comprise » est formulée sous forme d'injonctions concernant les outils à utiliser: "il faut utiliser le sabot », « on ne doit pas réintégrer le courrier mécanisé dans le casier ». Seuls deux documents nous permettent de retrouver la procédure de fusion prescrite: le premier est une affiche exposée sur le site 8 mois avant le changement, le second est un film d'environ 5 minutes présenté aux facteurs 2 mois avant le changement Ces documents présentent trois «techniques de fusion » différentes. L'application de l'une ou de l'autre de ces techniques est fonction de la façon dont le changement sera conduit sur le site (ordre d'introduction des nouveaux dispositifs techniques). Sur le site étudié, où l'ensemble des dispositifs a été introduit en même temps, la technique applicable (technique 2) comprend deux versions, en fonction du rendement de la machine. Le principe est de toujours fusionner le lot le moins important dans le lot le plus important. Si la machine trie plus de $50 \%$ du courrier du facteur, la procédure indique

2. Trois autres stratégies ont été observées de façon très minoritaire.

3. Un facteur de secteur est un facteur expérimenté qui remplace ses collègues et travaille donc sur plusieurs tournées. 
alors d'appliquer la «technique $2 \mathrm{VI}$ », qui consiste à insérer le courrier trié manuellement, dans le courrier trié mécaniquement préalablement disposé dans le sabot. Si la machine trie moins de $50 \%$ de la sacoche du facteur, c'est la «technique 2 V2 » qui est applicable. Il s'agit cette fois d'insérer le courrier trié par la machine, dans le courrier trié à la main préalablement " décasé » et disposé dans le sabot. Il est enfin mentionné qu'il ne faut «jamais recaser le courrier mécanisé ». Dans les documents transmis aux facteurs comme dans les données issues des entretiens avec l'encadrement et la direction de l'établissement, les justifications de cette procédure sont toujours évoquées dans une logique qui va de la procédure aux conséquences de sa non application (par exemple: «il est très important d'acquérir rapidement la méthode, et la bonne méthode de fusion, car celle-ci sinon, neutraliserait l'effet positif de la [machine] »). Les raisons de cette prescription y sont, de manières indirectes, portées par l'objectif global de la réorganisation qui est d' "optimiser le process de traitement et de distribution » du courrier. Mais le lien direct entre la procédure de fusion et la performance du processus n'est pas explicité. Les études menées au niveau national sur «les innovations technologiques » (conception, tests, études ergonomiques, calcul des cadences...) que nous avons pu recueillir auprès du médecin de prévention, du chef d'établissement et de l'organisateur du travail, traitent indépendamment du casier ou de la machine. La fusion, à l'interface des deux, semble absente des analyses réalisées.

Ainsi, les informations et formations transmises aux facteurs nous apparaissent comme des notions «ni théoriques, ni pratiques » (Mayen \& Savoyant, 1999) car elles ne nous permettent pas d'accéder $\mathrm{au}$ « sens » de cette procédure. D'une part, elles n'explicitent pas les raisons qui fondent l'interdiction de « recaser » le courrier mécanisé et qui prônent l'utilisation du sabot: les éléments qui permettent de comprendre l'élaboration de cette procédure, probablement issus d'une théorie « du flux de traitement du courrier », sont absents. D'autre part, la procédure n'apparaît pas pratique « au sens où elle ne réfère guère à l'activité en situation » (Mayen \& Savoyant, 1999). Les premières informations ont été transmises alors qu'il était envisagé de mettre en place un tri alphanumérique, bouleversement majeur dans l'activité du facteur qui trie depuis toujours dans l'ordre géographique de sa tournée. Puis la « technique 2 » applicable sur le site étudié a été présentée avec d'autres techniques, valables pour d'autres sites, à un moment ou ni la machine ni les casiers n'étaient présents. Enfin, la version alternative (technique 2 V2) n'a été que très brièvement évoquée. Pourtant, vu le rendement de la machine dans les premiers mois après le changement (entre 30 et $45 \%$ du courrier mécanisé), c'est normalement celle-ci qui aurait dû être appliquée.

En s'appuyant sur les résultats des recherches menées par Mayen et Savoyant (1999) à propos des règles de sécurité, nous pouvons dire que le contenu « ni théorique, ni pratique » de cette procédure prescrite n'a pas facilité sa compréhension. Or « une mauvaise compréhension des conditions d'application des procédures rend précisément cette application non seulement peu justifiée et porteuse de peu de significations mais aussi tout simplement beaucoup plus difficile (comme elle rend aussi plus difficile leur acquisition et leur mémorisation en cours de formation) » (Mayen \& Savoyant, 1999, p.85). L'appropriation (ou la non appropriation) d'une stratégie opératoire de fusion apparait donc liée au contenu des informations et formations divulguées aux opérateurs au cours du changement. La question est désormais de comprendre ce qui peut expliquer le développement des deux principales stratégies opératoires observées sur le site.

\section{2.- Appropriation d'une stratégie de fusion}

\subsection{1.- L'appropriation est fonction d'objectifs individuels de performance}

Les différents entretiens révèlent tout d'abord que les modalités d'appropriation d'une stratégie opératoire pour réaliser la fusion, répondent à des objectifs de performance (efficacité et efficience) que se fixent les facteurs. Ces objectifs peuvent être présentés selon les trois pôles de l'activité de travail fréquemment définis en ergonomie : le pôle système, le pôle soi et le pôle autres (Clot, 1995; Weill-Fassina \& Teiger, 1998, Weill-Fassina \& Pastré, 2004). Les objectifs définis dans le pôle « Sys- 
tème » regroupent les objectifs de l'entreprise et les critères d'efficacité productive (délai, qualité, quantité...). Dans cette catégorie, un premier objectif cité par les facteurs est celui de « gagner du temps », soit directement lors du tri, soit en anticipant dès le tri la phase de distribution. Un second objectif est celui d'effectuer une tournée de qualité, c'est-à-dire sans erreurs et sans laisser de lettres de côté. Les objectifs définis selon le pôle « Soi » font référence aux aspects personnels de l'activité. Il peut s'agir d'objectifs de préservation de sa santé ou d'une volonté de réaliser son activité selon « sa propre conception du métier». Car le sens que met chaque facteur derrière l'idée d' "une tournée bien faite » lui est généralement bien spécifique, « depuis une tournée la plus rapide possible, bien à l'heure, où on jette le courrier au client, à l'américaine » jusqu'au "service rendu à l'usager, individuellement, pour lui faire plaisir $»$. Enfin, des objectifs individuels de performance sont définis par rapport aux « Autres » c'est-à-dire par rapport à l'équipe, à la hiérarchie ou à d'autres centres décisionnaires de l'entreprise. Ces objectifs peuvent être déclinés en terme de comparaison aux autres membres de l'équipe (ne pas vouloir finir en dernier ou en premier par exemple) ou en fonction de l'impact de son activé sur le travail de ses collègues (ne pas vouloir faire attendre ses collègues par exemple). Il peut s'agir aussi de vouloir respecter la procédure recommandée par la hiérarchie.

La poursuite de ces différents objectifs relève de modalités personnelles et chaque objectif a un impact complexe sur l'appropriation de l'une ou de l'autre des deux stratégies. Par exemple, si tous les facteurs s'accordent à chercher à gagner du temps directement lors de la phase de tri, pour certain "forcer, écarter les intercalaires » du casier pour y introduire le courrier est trop long et il vaut mieux utiliser le sabot, pour d'autre, utiliser le sabot rajoute une "reprise du courrier» i.e. une « manipulation supplémentaire » et le choix de réinsérer les plis dans le casier "économise donc le temps». De même, pour anticiper pendant la phase de tri la distribution du courrier, il apparaît pour beaucoup préférable d'utiliser le sabot: cela permet d'avoir « une meilleure vision globale de sa tournée, de mieux mémoriser » puisqu'en effet les lettres triées manuellement sont, avec cette stratégie, lues deux fois et le lot fusionné est visionné dans sa globalité. «Avec la technique du sabot, je maîtrise mieux la distribution, je sais avant de partir, ou je vais devoir m'arrêter ». Mais l'utilisation du casier, quand à elle, autorise une rectification des erreurs jusqu'à la fin de la phase de tri, et en particulier, elle permet de «mieux rattraper les erreurs de la machine ». En effet, alors qu' avec l'utilisation du sabot le courrier est positionné dans les caissettes ou « botté » (i.e. liassé) au fur et mesure du tri, l'utilisation du casier laisse accessible l'ensemble de la tournée jusqu'au « décasage » juste avant le départ: «Avec le casier, si je vois une erreur dans le tri «méca », ou si y a des "fausses » [lettres mal classées qui se retrouvent parfois sur d'autres positions] c'est facile je reviens en arrière ». Un autre exemple peut être donné par rapport à l'objectif de préservation de sa santé: un des avantages de la stratégie du sabot par exemple est de pouvoir s'asseoir, «c'est quand même plus agréable, c'est du confort, et puis c'est aussi mieux pour le dos». Mais pour d'autres, il est préférable d'être «carrément debout, plutôt que d'avoir à se lever régulièrement. »

Ces différents exemples montrent que durant la phase de tri, les facteurs poursuivent une diversité d'objectifs de performance (i.e. d'efficacité et d'efficience) qui sont avant tout personnels, plus ou moins partagés par l'équipe mais dont la combinaison et la hiérarchisation est propre à chacun. En outre la poursuite de ces différents objectifs joue un rôle sur les modalités d'appropriations d'une stratégie de fusion.

\subsection{2.- L'appropriation est fonction des exigences de chaque situation de travail}

En plus d'objectifs de performance, l'appropriation d'une stratégie lors de la fusion est liée aux caractéristiques de chaque situation de travail qui constituent des contraintes ou des ressources dans le développement de l'activité. Cette fois encore, les modalités d'appropriation peuvent être regroupées selon les trois pôles de l'activité. Le pôle «Système » regroupe des exigences liées à la quantité de courrier à traiter, au type de tournée réalisée par le facteur (tournée « ville » ou « campagne », moyen de locomotion utilisé, présence de «bis/ter »...) ou au rendement de la machine. Par exemple, une «tournée voiture » se charge, en plus du courrier, de colis et effectue des distances plus longues 
(« tournée en campagne ») ce qui signifie que le facteur a relativement moins de plis à trier. À l'inverse, une «tournée scooter » comporte d'avantage de points de distribution, plus rapprochés et ne comprend pas la distribution des colis. Le nombre de plis à traiter est donc plus important et bien souvent, le nombre de points de distribution est supérieur au nombre de cases du casier. «Beaucoup de cases doivent donc être doublées, voir triplées », ce qui signifie qu'il reste une étape de tri à réaliser au sein de ces cases. Dans ce cas l'utilisation du sabot est plus adéquate. Cette relation entre les caractéristiques de la tournée et l'appropriation d'une stratégie de fusion a pu être observée sur le terrain. Par exemple, les stratégies utilisées par deux facteurs de secteur ${ }^{4}$ varient en fonction de la tournée sur laquelle ils sont affectés. "Au départ, j'ai utilisé le sabot, comme on nous avait dit... mais il a des tournées, c'est clair, tu as juste une caissette, tu prends 10 minutes à remettre dans les cases... Pour, la $n^{\circ} \mathrm{X}$, là c'est sûr, je fusionne [avec le sabot]: elle a beaucoup de tri et les numéros se suivent. Mais par exemple, pour la $n^{\circ} Y$, avec tout ses "tricotages ${ }^{5} »$, même si elle est chargée, je préfère le casier ».

Les caractéristiques liées au pôle « soi » regroupent les caractéristiques physiques de chacun ainsi que les compétences et les stratégies développées avec l'expérience. En voici trois exemples:

— «Être de petite taille fait que l'on ne peut pas rester assis même si on fusionne [avec le sabot] »,

- Avoir besoin d'insérer des « cartons de réexpédition [cartons glissés dans les cases afin de repérer les lettres à réexpédier] pour mémoriser et visualiser les lettres à réexpédier » s'oppose à la stratégie du casier puisque ces «cartons remplissent les cases et qu'il est nécessaire de les retirer avant de botter »,

- Utiliser la « combine du marque-page $e^{6}$ n'est pas possible avec la stratégie du casier. Vouloir conserver cette stratégie "qui s'apprend avec le métier » devient alors une contrainte favorisant l'appropriation de la stratégie du sabot.

Enfin, les « Autres» constituent aussi des caractéristiques spécifiques à chaque situation de travail. Dans le centre de tri, chaque facteur sait comment « fusionnent» ses « voisins de casier » ( Sur la $Y$, à côté, elle fusionne dans le casier alors qu'elle est en scooter », " mais X, lui il change parfois de technique ») et bénéficient des critiques et conseils des collègues proches ( $m o i$, à ta place, j’utiliserai même 2 billots, pour maintenir les 2 lots.» «Sacré cadence! »). Ces échanges et les exemples des opérateurs voisins constituent des ressources dans le développement d'une stratégie de fusion ( $O n$ a regardé les autres et on s'est rendu compte qu'ils semblent être plus rapides alors, autant tester de faire comme eux »). Des responsables hiérarchiques ont, à l'inverse, contraint quelques facteurs en leur « conseillant fortement » de suivre la prescription.

Ainsi, l'appropriation d'une technique est non seulement fonction d'une diversité d'objectifs poursuivis par les facteurs mais aussi d'une grande variété de contraintes et de ressources liées aux caractéristiques de la tournée, à chaque individu, à chaque moment de production c'est-à-dire aux exigences de chacune des situations de travail. Le schéma ci-dessous (cf. Figure 1.) récapitule les différentes modalités d'appropriation d'une stratégie opératoire lors de la fusion. Aucune de ces modalités n'apparaît déterminante dans l'appropriation d'une stratégie opératoire, et pour chaque facteur, le développement de l'un, de l'autre, ou des deux instruments dépend d'une combinaison singulière et évolutive de ces modalités. Car ces modalités ne sont pas stables dans le temps mais évoluent selon les spécificités de chaque situation de travail au jour le jour: la quantité de courrier et le rendement de la machine fluctuent, les objectifs de préservation de sa santé peuvent varier, les règles imposées ou les conseils donnés par les encadrants de proximité ne sont pas toujours identiques, les collègues qui distribuent le courrier en aval ne sont pas toujours les mêmes etc. Beaucoup de facteurs ont d'ailleurs

4. Les facteurs de secteurs sont des facteurs expérimentés qui travaillent en remplacement sur plusieurs tournées.

5. Les rues « tricotées » sont les rues pour lesquelles le facteur passe d'un coté de la rue à l'autre, en alternant donc des $\mathrm{n}^{\circ}$ pairs et impairs qui sont souvent décalés et donc ne se suivent plus.

6. La stratégie « du marque-page » consiste à utiliser des « grands formats » (le quotidien local généralement) ou à « retourner des lettres » dans le sens inverse de la botte pour séparer les points de distribution dans les lots triés et ainsi faciliter le dépôt des lettres durant la tournée. 
«essayé les deux » et «changé de technique » plusieurs fois. Dans les discours, aucune des deux stratégies (sabot ou casier) ne semble devoir s'imposer sur l'autre: chacune porte "ses avantages et ses inconvénients », la «bonne méthode, c'est personnel, à chacun sa cadence, à chacun son petit confort, à chacun sa tournée ». "La stratégie de fusion, c'est individuel ».

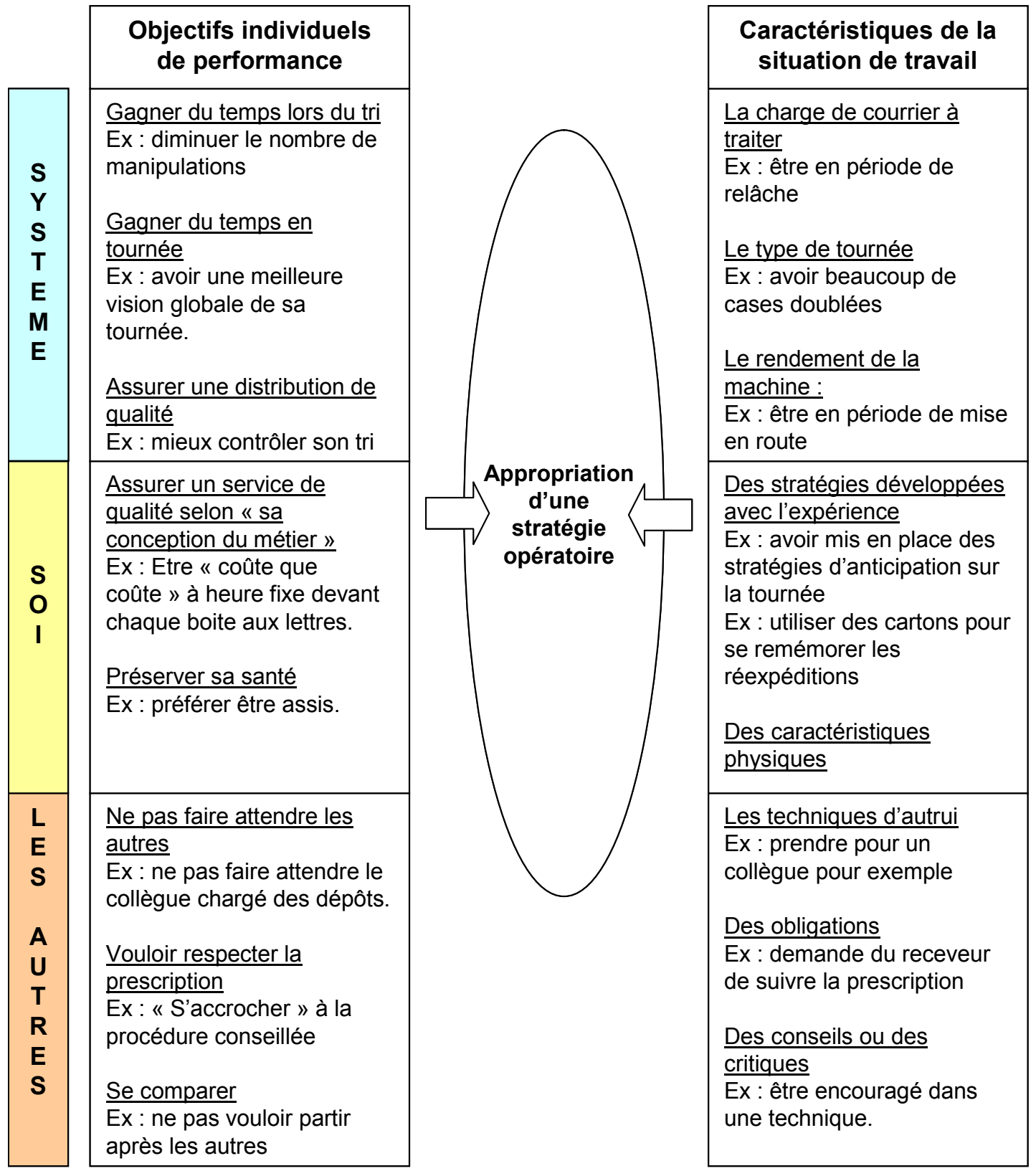

Figure 1: Modalités d'appropriation d'une stratégie opératoire lors de la fusion

Figure 1: Terms of development of an operative strategy for sorting post 


\subsection{3.- L'appropriation est collective}

D'après les résultats précédents, «la stratégie de fusion est individuelle », c'est-à-dire est fonction de caractéristiques spécifiques à chaque situation de travail et d'objectifs de performance individuels, dont la définition et la combinaison sont propres à chaque opérateur, à chaque moment de production. On pourrait alors s'attendre à voir s'installer dans la durée ces deux stratégies opératoires, selon une certaine proportion, éventuellement variable. Mais l'analyse de l'évolution des stratégies opératoires employées montre, qu'au fil du temps, la «stratégie du casier » s'impose sur le site (cf. figure 2).

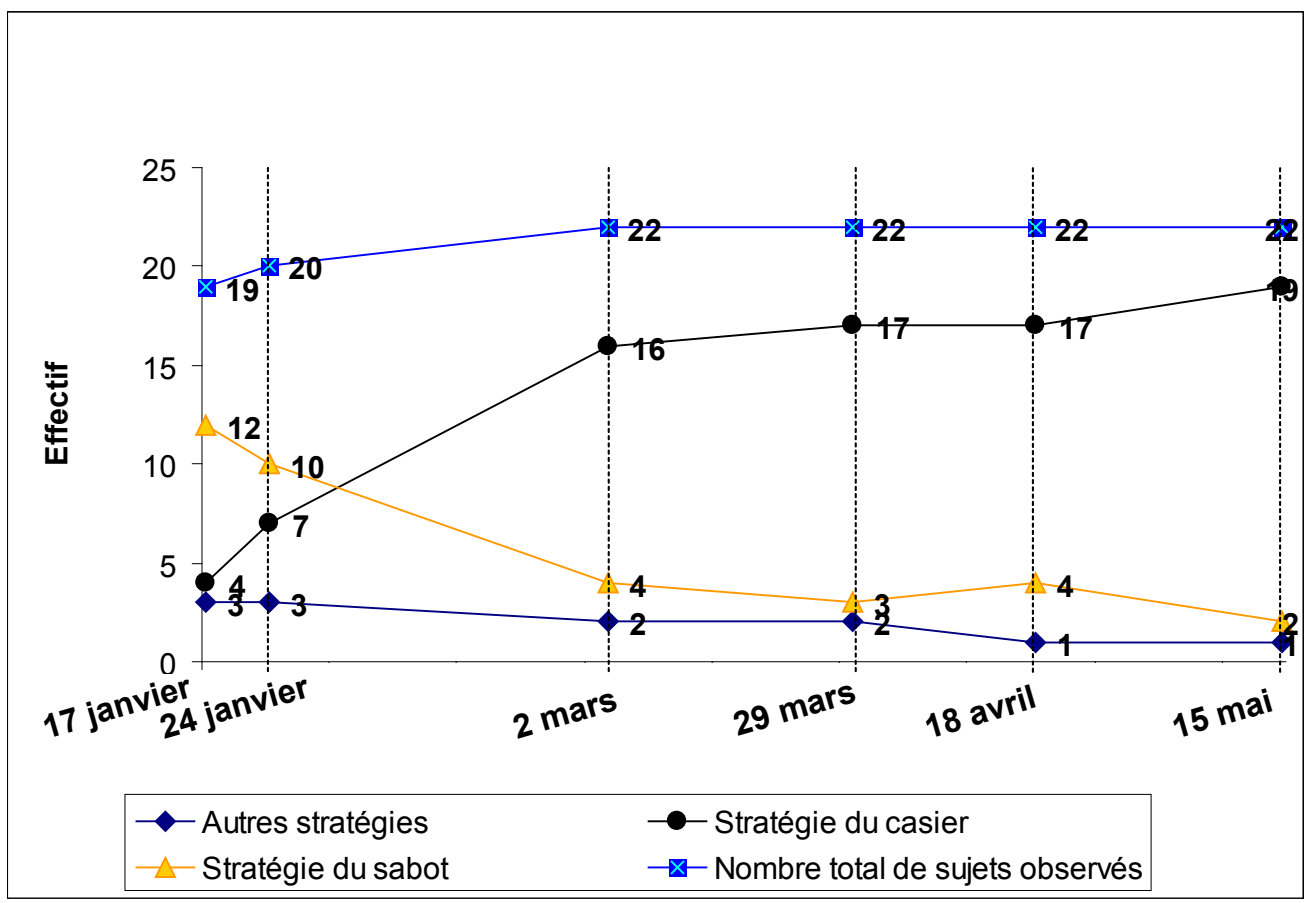

Figure 2: Evolution des stratégies opératoires développées sur le site entre janvier et juin 2007.

Figure 2: Changes in operative strategies developed between January and June 2007.

Durant les entretiens, les facteurs, ayant changé « définitivement » de stratégie précisent que ceci ne «s'est pas fait à un moment précis », que cela s'est " généralisé progressivement ». Les références aux dimensions collectives sont nombreuses («Maintenant, je fais comme tout le monde », « Ah, ça y est tu fais comme nous! »). Et, tout au long de l'étude, les facteurs ont cherché à se renseigner sur la prépondérance des deux stratégies développées dans l'équipe: «Je voudrais bien savoir le nombre qui se serve du [sabot]... » «On est plus nombreux à faire comme ça maintenant, non? ». En effet, parmi les modalités d'appropriation précédemment listées, la dimension « autre » occupe une place primordiale: dans cette période de changement, les résultats des entretiens montrent que le principal critère d'évaluation de la performance d'une stratégie par les facteurs est le fait que cette stratégie soit partagée. Pour qu' « une technique » soit jugée «perfomante », il «faut qu'elle soit partagée par tous », «que tout le monde la fasse », «que l'on se sente tous unis».

De même, le sens et la définition de cette nouvelle étape de tri ont fait l'objet de négociations au sein du groupe. Car en effet, durant les premiers mois, le mot « fusion » est resté un mot ambigu, polysémique. Parfois il faisait référence à l'action d'intégrer les deux lots, mécanisé et manuel, en un seul. Par exemple: "Il y a plusieurs manières de fusionner, avec le sabot ou avec le casier » ou bien: «La fusion, je ne la fais comme elle devrait être faite, je réinsère les plis dans les cases ». Mais, beaucoup plus fréquemment, le terme « fusion » signifiait uniquement «la bonne méthode », la tâche 
« comprise » par les facteurs (Veyrac Merad-Boudia, 1998), c'est-à-dire uniquement l'utilisation du sabot: «la fusion, je ne la fais pas, j'insère directement dans les cases » ou alors « avec les CIDEX', on n'est pas obligé de fusionner, on a plus de place pour remettre dans les cases ». Au fil du temps, il fut de plus en plus fréquent de voir les facteurs se reprendre et s'accorder sur la première définition de ce « mot problématique » (Carboni, 2001), et par là même, tenter de légitimer au sein du groupe, que le fait de «fusionner, c'est aussi utiliser la stratégie du casier»:

— «Mais là aussi tu fusionnes, mais pas avec la même technique, pas avec celle qu'on devrait faire»

— «Mais, là c'est quand même de la fusion ce qu'on fait? - Bah oui, puisque tu incorpores ce lotlà avec l'autre »

— « Les deux [techniques] sont de la fusion... parce que en fait la fusion, c'est mettre ensemble les deux lots, c'est tout. »

Il en fut de même à propos du sens de cette étape de «fusion », de ses objectifs et des raisons qui ont conduit la direction à prescrire cette procédure. Tout au long de l'étude, les facteurs ont cherché à définir collectivement un but, « une théorie », derrière la procédure conçue (Mayen \& Savoyant, 1999):

— «À cause de la largeur des cases, c'est vite à côté et il est impossible de revenir en arrière... ça doit être pour ça qu'il nous demande de fusionner normalement, tu crois pas?»

— «La fusion a sûrement été faite pour récupérer les erreurs, et c'est pour ça qu'on doit être plus attentif avec le casier, pour être bon du premier coup...»

Ainsi, à travers cet accord sur la stratégie opératoire à employer lors de la fusion, sur une clarification commune de son sens et de sa définition, les facteurs définissent des «obligations sur lesquelles s'entendre pour arriver à travailler ensemble » (Clot, 2000, p.278). L'appropriation d'une stratégie répond donc certes, à des modalités individuelles, mais s'insère aussi dans la reconstruction ou le maintien du collectif de travail. Il s'agit en effet de (re)définir, autour du développement de cet instrument, des règles de métiers, "qui articulent l'organisation du travail-qu'elles complètent voire qu'elles corrigent - et l'engagement subjectif de chacun, qu'elles appellent » (Cru, 1995). Et la distance avec ces règles de métier, avec ce «genre professionnel », c'est-à-dire l'appropriation d'une stratégie opératoire singulière, ne pourra éventuellement se faire qu'une fois ce collectif stabilisé. Car « la non maîtrise du genre et de ses variantes interdit l'élaboration du style. Prendre des libertés avec les genres implique une grande maîtrise de ces derniers »(Clot, 2000, p.278). Ainsi, si «la stratégie de fusion est individuelle », son appropriation apparaît, quant à elle collective.

\section{Discussion}

D'autres résultats issus de cette recherche montrent que ce changement fut une période au cours de laquelle les opérateurs ont été fragilisés. Cette étape d'appropriation et d'apprentissage, durant laquelle de nouvelles compétences et de nouveaux instruments ont dû être développés sur la base d'artefacts matériels et symboliques, a en effet été vécue comme « une épreuve » "difficile » et « fatigante » (Cuvelier, 2007). D'autant plus que la fragilisation a aussi concerné les dimensions collectives du travail: les formes de réalisation du travail collectif visant entre autre des régulations et des entraides entre collègues ont été altérées (Cuvelier \& Caroly, 2008). L'appropriation de stratégies opératoires par l'équipe s'insère nécessairement dans cette phase de fragilisation. Elle s'effectue selon des modalités diverses, fonction à la fois des exigences de chaque situation de travail et des objectifs de performance fixés par chacun, mais aussi en lien étroit avec le maintien du collectif de travail. La façon dont s'articulent et se combinent les différentes modalités d'appropriation listées n'a pas pu être mise à jour, car ces combinaisons sont singulières et évolutives, propres à chaque in-

7. «Courrier Individuel à Distribution Exceptionnelle ». Cet acronyme est employé pour désigner des boîtes aux lettres de particulier regroupées en un lieu unique. 
dividu et à chaque moment de production. Reconstruire la dynamique individuelle de ces modalités aurait nécessité la réalisation d'entretiens individuels bien plus fréquents avec les facteurs durant cette période de changement. Ceci n'était pas envisageable dans le contexte du terrain et constitue une limite de l'étude. Mais, si plusieurs stratégies opératoires ont été explorées au sein de l'équipe selon ces modalités, les résultats révèlent que le maintien d'un « genre professionnel » a favorisé l'appropriation collective d'une stratégie opératoire commune.

Or cette stratégie opératoire ne correspond pas à la stratégie prévue et prescrite par les concepteurs ${ }^{8}$. «Pire », c'est une « stratégie interdite» « qui neutralise les effets de la machine de tri TTF ». Et le débat au sein de la hiérarchie se cristallise autour du «non-respect des procédures », de la «mauvaise compréhension des nouvelles règles industrielles » et des comportements « de résistance » des opérateurs. Pour la direction et l'organisation du travail, il s'agit désormais que ces derniers acquièrent « le bon geste » et appliquent la « bonne technique ». On devine alors un enchaînement possible vers la mise en place d'un mode de management plus ferme et des prescriptions plus fortes entraînant un renforcement des contraintes. Il est bien sûr préférable de repartir de la compréhension des raisons pour lesquelles les opérateurs « font autrement» et utilisent « non convenablement» ce nouvel outil de tri. «Autrement dit, il est plus pertinent de partir de l'hypothèse que les gestes, et plus généralement les stratégies opératoires auxquels les opérateurs ont recours sont les meilleurs compromis trouvés par eux, à un moment donné » (Bourgeois \& al., 2006, p.88) et de laisser ouverte la gamme des développements possibles. Le maintien du collectif de travail, ressource potentielle pour la santé du sujet qui travaille (Caroly \& Clot, 2004), est un élément qu'il ne faut pas négliger dans la mise en œuvre de ces compromis. En imposant à certains, « ayant trop de retard » d'appliquer la stratégie prescrite, le risque est de «briser ce qui fait la cohésion si fragile et si chèrement acquise du collectif de travail » (Dejours, Dessors, \& Molinier, 1994).

En tout état de cause, pour qu'une réforme technique puisse avoir des chances de succès, il faut qu'elle ait deux qualités :

- Qu'elle fasse évoluer tout le collectif de travailleurs ensemble, faute de quoi la coopération entre eux serait ruinée;

- Qu'elle permette l'éclosion d'un nouveau système de valeur et d'un nouveau code gestuel dans les techniques proposées, sans quoi les ouvriers y perdent les moyens de reconnaître leur rapport d'appartenance à la communauté »(Op. Cit, p.7).

Par ailleurs, cette étude interroge la notion de conception. En ergonomie, trois grandes approches ont successivement été proposées pour rendre compte de l'activité future dès la conception (Falzon, 2005; Béguin \& Cerf, 2004). La première est l'approche la plus classique qui vise à anticiper l'activité future et à ramener les usages dans la conception. La seconde stipule que la conception se poursuit dans l'usage et vise donc à concevoir des systèmes plus «souples », ou «flexibles », qui intègrent des marges de manœuvre pour le développement de l'activité future (Daniellou, 2004). En ce sens, on peut supposer que le nouveau casier de tri ait été conçu selon ce mode de conception, puisque le caractère modulable de cet outil permet au facteur titulaire de modifier et d'adapter son casier en fonction de sa tournée, de le faire évoluer dans le temps selon, par exemple, l'évolution démographique, l'évolution de la production ou selon ses préférences. La dernière approche est l'approche développementale nommée « conception distribuée » (Béguin, 2005). Elle consiste à assurer un développement conjoint des situations et des personnes, c'est-à-dire à intégrer le développement des activités dans les processus conception. Autrement dit, la « conception distribuée » cherche à «articuler dans un même mouvement, le développement des situations (artefact et/ou organisation) par les concepteurs et le développement des ressources de leur action par les opérateurs » (Béguin \& Cerf, 2004, p.61). Elle est appréhendée comme un «processus d'apprentissages mutuels »entre les concepteurs et les utilisateurs (Béguin, 2007). Concrètement, ces processus d'apprentissages

8. Le terme « concepteurs » est employé au sens large d' « acteurs de la conception ». Il ne désigne pas uniquement les « concepteurs professionnels » mais aussi d'autres catégories professionnelles impliquées dans la conduite de projet (organisateurs du travail, chargés d'étude sur la santé, responsables en communication, etc.) (Béguin \& Cerf, 2004). 
nécessitent la mise en place de démarches participatives permettant des interactions entre usagers et concepteurs. Et nous savons que la mise en œuvre de ces actions de conception participative soulève des questions qui «sont loin d'être triviales »: «objectifs assignés aux opérateurs, choix des représentants des utilisateurs, construction des méthodes participatives...» (Darses \& Reuzeau, 2004, p.409). Les résultats de notre recherche interrogent aussi cette mise en œuvre. Car si le but de la conception est d'articuler dans un même cadre le développement des ressources et celui de l'artefact et que, le développement des ressources «suppose une dimension plus large de l'activité », qui prenne notamment en compte «les formes organisées de l'action au sein des collectifs » (Béguin, 2007) - ce que tend à montrer notre recherche - nous pouvons nous interroger sur les modalités d'articulation possibles entre le développement des situations de travail et celui des collectifs de travail dans les processus de conception. L'échantillon d'opérateurs sélectionnés pour participer à la démarche peut-il rendre compte des dynamiques collectives de l'équipe ? Et ce d'autant plus si la conception se situe à l'échelle d'un grand groupe au sein duquel il est démontré que les formes de réalisation du travail collectif diffèrent d'un établissement à un autre (Flageul-Caroly, 2001). En effet, « il semblerait que l'importation stricte d'un outil donnant satisfaction, conçu ailleurs dans un cadre participatif, ne permettrait pas d'atteindre d'aussi bons résultats » (Coutarel, 2004, p.362). L'appropriation d'une stratégie opératoire est un processus continu de développement, qui dépend des caractéristiques et des objectifs de chaque situation de travail, et s'articule avec le développement du collectif de travail.

\section{REMERCIEMENTS}

Nous tenons à remercier l'équipe du centre de tri et de distribution du courrier qui nous a accueillies, et tout particulièrement la médecin de prévention professionnelle avec qui nous avons étroitement collaboré durant cette étude.

\section{RÉFÉRENCES}

Béguin, P. (2005). Concevoir pour les genèses professionnelles. In P. Rabardel, \& P. Pastré (Eds.), Modèle du sujet pour la conception. Dialectiques activités développement (pp. 32-52). Toulouse: Octarès Editions.

Béguin, P. (2007). Innovation et cadre socio-cognitif des interactions concepteurs-opérateurs: une approche développementale. Le travail humain, 4(70), 369-390.

Béguin, P., \& Cerf, M. (2004). Formes et enjeux de l'analyse de l'activité pour la conception de systèmes de travail.@ctivités, 1(1), 54-71.

Benchekroun, T.H., \& Weill-Fassina, A. (2000). Le travail collectif. Perspectives actuelles en ergonomie. Toulouse: Octarès Editions.

Bernoux, P. (2002). Le changement dans les organisations entre structures et interactions. Relations industrielles / Industrial relations, 57(1), 77-99.

Bernoux, P. (2004). Sociologie du changement dans les entreprises et les organisations. Paris: Editions du Seuil.

Bourgeois, F., Lemarchand, C., Hubault, F., Brun, C., Polin, A.,. Faucheux, J.M., Douillet, P., \& Albert, E. (2006). Troubles musculosquelettiques et travail. Quand la santé interroge l'organisation. Lyon: ANACT.

Carboni, P. (2001). L'activité réflexive chez les facteurs de la distribution postale. Education permanente, $146,59-71$.

Caroly, S. (2002). « Gérer les règles et le client » : implication des cadres dans les modalités d'ajustement des règles pour une organisation efficiente. In Les évolutions de la prescription, Actes du 37ème Congrès de la Société d'Ergonomie de Langue Française. Aix-en-Provence (pp. 276-284).

Caroly, S., \& Clot, Y. (2004). Du travail collectif ai collectif de travail : développer des stratégies d'expérience. Formation Emploi, n88, 43-55. 
Chassaing, K. (2004). Vers une compréhension de la construction des gestuelles avec l'expérience: le cas des « tôliers » d'une entreprise automobile. Pistes, 6(1).

Chassaing, K. (2005). Stratégies d'expérience et organisation du travail dans la prévention des douleurs articulaires. Actes du $1^{\text {er }}$ congrès francophone sur les TMS du membre supérieur. Nancy.

Clot, Y (1995). La compétence en cours d'activité. Education Permanente, 123(2), 115-123

Clot, Y. (1999). Le geste est-il transmissible? Actes du 10e entretiens de la Villette : Apprendre autrement aujourd'hui? Paris.

Clot, Y. (2000). La fonction psychologique du collectif. In T.H. Benchekroun, \& A. Weill-Fassina (Eds.), Le travail collectif. Perspectives actuelles en ergonomie (pp. 272-286). Toulouse: Octarès Editions.

Clot, Y. (2004). La fonction psychologique du travail (4 $4^{\mathrm{eme}}$ édition corrigée). Paris: PUF.

Clot, Y., \& Fernandez, G. (2005). Analyse psychologique du mouvement: apport à la compréhension des TMS. @ctivités, 2(2),68-78.

Clot,Y., Scheller, L., Caroly, S., Millanvoye, M., \& Volkoff, S. (2000). Le travail comme genre professionnel comme contribution à la genèse de l'aptitude. Paris: Collection de la mission de la Recherche de La Poste.

Coutarel, F. (2004). La prévention des troubles musculo-squelettiques en conception: quelles marges de manoeuvre pour le déploiement de l'activité ? Thèse de doctorat en ergonomie, Université Victor Segalen Bordeaux 2, LESC.

Cru, D. (1988). Collectif et travail de métier. In C. Dejours (Ed.), Plaisir et souffrance dans le travail (pp. 43-49). Paris: Edition de l'AOCIP.

Cru, D. (1995). Règle de métier. Langue de métier. Diplôme d'ergonomie, Paris: Ecole Pratique des Hautes Etudes.

Cuvelier, L. (2007). Changement organisationnel et technique : étude de l'appropriation d'une stratégie opératoire par un collectif de travail. Mémoire de Master recherche en Ergonomie. Paris: Cnam..

Cuvelier, L., \& Caroly, S. (2008). Conception et appropriation d'un nouveau casier de tri. In P. Negroni, \& Y. Haradji (Eds.), Actes du 43ème congrès de la SELF (pp. 54-60). Editions ANACT.

Daniellou, F. (2004). L'ergonomie dans la conduite de projets de conception de systèmes de travail. In P. Falzon (Ed.), L'ergonomie (pp. 359-373). Paris: PUF.

Darses, F., \& Reuzeau, F. (2004). Participation des utilisateurs à la conception des systèmes et dispositifs de travail. In Falzon, P. (Ed.), Ergonomie (pp. 405-420). Paris: PUF.

De la Garza, C., \& Weill-Fassina, A. (2000). Régulations horizontales et verticales du risque. In T. H. Benchekroun, \& A. Weill-Fassina (Eds.), Le travail collectif. Perspectives actuelles en ergonomie (pp. 217-224). Toulouse: Octarès Editions.

Dejours, C., Dessors, D., \& Molinier, P. (1994). Documents pour le médecin du travail, n58, pp.112-117, Ed. INRS \& Ministère du Travail.

Falzon, P. (2005). Ergonomie, conception et développement. Conférence introductive, $40^{\text {ème }}$ Congrès de la SELF, Saint-Denis, La réunion.

Fernandez, G. (2001). Le corps, le collectif et le développement du métier. Etude clinique d'un geste de métier à la SNCF. Education Permanente, n¹46, 27-33.

Flageul-Caroly, S. (2001). Régulations individuelles et collective de situations critiques dans un secteur de service: le guichet de La Poste. Thèse de Doctorat d'Ergonomie. Paris: EPHE.

Folcher, V., \& Rabardel, P. (2004). Hommes, artefacts, activités : perspective instrumentale. In P. Falzon (Ed.), Ergonomie (pp. 251-268). Paris: PUF.

Gonzalès, R., Teiger, C., \& Weill-Fassina, A. (2002). Le serpent qui se mord la queue : Règles et activités de régulation lors de l'accueil en crèches collective. In Les évolutions de la prescription, Actes du 37ème Congrès de la Société d'Ergonomie de Langue Française. Aix-en-Provence, pp. 248-254. 
Gaudart, C., \& Pueyo, V. (2000). L'expérience dans les régulations individuelles et collectives de l'efficicence. In T.H. Benchekroun, \& A. Weill-Fassina (Eds.), Le travail collectif. Perspectives actuelles en ergonomie (pp. 257-271). Toulouse: Octarès Editions.

Gaudart, C., \& Weill-Fassina, A. (1999). L'évolution des compétences au cours de la vie professionnelle : une approche ergonomique. Formation Emploi, 67, 47-62.

Lacoste, M. (1992). Apprentissage dans le travail et interaction. Langage \& Travail, ${ }^{\circ} 3,35-42$.

Leplat, J., \& Hoc, J.-M. (1983). Tâche et activité dans 1'analyse psychologique des situations. Cahiers de psychologie cognitive, 3(1), 49-63.

Mayen, P., \& Savoyant, S. (1999). Application de procédure et compétences. Formation- Emploi, $\mathrm{n}^{\circ}$ 67, 77-92.

Mayen, P., \& Vidal-Gomez, C. (2005). Conception, formation et développement des règles au travail. In P. Rabardel, \& P. Pastré (Eds.), Modèle du sujet pour la conception. Dialectiques activités développement (pp.109-128). Toulouse: Octarès Editions.

Mollo, V., \& Falzon, P. (2004). Auto- and allo-confrontation as tools for reflective activities. Applied Ergonomics, 35(6), 531-540.

Rabardel, P. (1995). Les hommes et les technologies, approche cognitive des instruments contemporains. Paris: Armand Colin.

Rabardel, P. (2005). Instrument subjectif et développement du pouvoir d'agir. In P. Rabardel, \& P. Pastré (Eds.), Modèle du sujet pour la conception. Dialectiques activités développement (pp. 11-29). Toulouse: Octarès Editions.

Sardas, J.C. (1994). Comprendre et gérer les mutations organisationnelles : cohérences fonctionnelles et dynamiques des acteurs. Performances humaines \& techniques, $\mathrm{n}^{\circ}$ hors série, 30-38.

Schmidt, K. (1994). Coopérative work and its articulation: requirements for computer support. Le Travail Humain, 57(4), 345-366.

Veyrac Merad Boudia, H. (1998). Approche ergonomique des représentations de la tâche pour l'analyse d'utilisation de consignes dans des situations de travail à risques. Thèse de doctorat en ergonomie, Université Toulouse le Mirail.

Vidal-Gomel, C. (2002). Système d'instruments : un cadre pour analyser le rapport aux règles de sécurité. In Actes du 38ème Congrès de la SELF, Les évolutions de la prescription (pp. 134-144). Aix-en-Provence.

Weill-Fassina, A., \& Pastré, P. (2004). Les compétences professionnelles et leur développement. In P. Falzon (Ed.), Ergonomie (pp. 213-231). Paris: PUF.

Weill-Fassina, A., \& Teiger, C. (1998). Evolution des compétences professionnelles. Allocution lors d'un séminaire interne entre le Laboratoire d'Ergonomie du CNAM et le Laboratoire d'Ergonomie Physiologique et Cognitive de l'EPHE, Paris.

\section{RÉSUMÉ}

Cette recherche est basée sur le suivi d'un changement organisationnel et technique mené dans un centre de tri et de distribution du courrier. Son objectif est de comprendre comment les facteurs developpent leur activité dans le double contexte d'une organisation du travail modifiée et de l'implantation de nouveaux dispositifs techniques. Les travaux en sociologie et en didactique professionnelle nous ont conduit à aborder l'analyse ergonomique de ce changement à travers le concept d' « appropriation », décliné selon trois niveaux : l'appropriation des outils, l'appropriation des procédures et l'appropriation des gestes de travail. Le développement d'un instrument (à partir de l'artefact « casier de tri ») et les modalités d'appropriation d'une nouvelle stratégie opératoire (lors d'une phase spécifique du tri manuel du courrier nommée la fusion) sont plus spécifiquement analysés. Les résultats montrent que cette appropriation se 
fait selon plusieurs modalités, en fonction des caractéristiques de chaque situation de travail et des objectifs de performances poursuivis par les opérateurs, mais aussi selon des dimensions collectives du travail.

Mots-CLÉS

Appropriation, collectif de travail, changement, conception, développement.

\section{RÉFÉRENCEMENT}

Cuvelier, L. \& Caroly, S. (2009). Appropriation d'une stratégie opératoire: un enjeu du collectif de travail, Activités, 6 (2), pp. 57-74. http://www.activites.org/v6n2/v6n2.pdf

Article soumis le 15 janvier 2009 accepté pour publication le 17 juillet 2009 